\title{
Some Prospects for Chemical Analysis
}

Yu A Zolotov ${ }^{1,2 *}$

${ }^{1}$ Department of Chemistry, Lomonosov Moscow State University, Russia

${ }^{2}$ Kurnakov Institute of General and Inorganic Chemistry, Russian Academy of Sciences, Russia

\begin{abstract}
Needs in chemical analysis were considered, for example requirements to "field", speciation analysis, distribution (local), remote, non-destructive analysis, miniaturization, and automatization. General approaches to analysis were discussed. Search and studies of novel phenomena, regularities and properties of substances which can serve as a basis of new techniques and tools of analysis were noted as significant directions for the future. Assumption that multistep analysis with screening at the first steps will be used more widely was made (metrology of chemical analysis and related fields will be also developed). Prospects of main analytical techniques were estimated (spectroscopy, mass spectrometry, chromatography, and electrochemistry). Priorities in objects to be analyzed and important analytes were mentioned.
\end{abstract}

\section{Keywords}

Analytical chemistry, Green analytical chemistry, Chemical analysis, Analytical techniques, Objects to be analyzed

\section{Introduction}

In the last years, many articles partially affecting perspectives of various fields of analytical chemistry have been published, for instance on analytical application of ionic liquids [1], process analytical chemistry [2,3], sample preparation [4-6], aptamers in analytical chemistry [7], surface enhanced Raman spectroscopy (SERS) in microbiology [8], solid-state electrochemistry [9], LIBS in archeological science [10]. Consideration of prospects for various analytical techniques and applied areas has been made in reviews of TrAC-Trends in Analytical Chemistry, for example on combination of chemical and biological methods for integrated environmental assessment [11]. However, publications devoted to attempts to estimate the future development of analytical chemistry as a whole were not found. This article can be considered as an expansion and up-to-dating of the previous short editorial [12]. Prospects for the development of analytical chemistry for the next, say, 10-15-years can be considered in several directions: 1) needs, hence goals, and problems; 2) general approaches to chemical analysis; 3 ) analytical techniques and tools and 4) main objects of analysis and basic analytes.

\section{Needs, Hence Goals and Problems}

We can assume further movement of chemical analysis from a stationary laboratory to the place, where the object to be analyzed is located. The development and widespread use of out-of-laboratory analysis is caused by significant demand. During "field" analysis, the need for transportation and storage of samples disappears, but more importantly that many samples can only be analyzed on-site-in particular, because of their lability, toxicity, or the need to provide an urgent study
[13]. Relatively easy-to-use means of monitoring environmental objects, express analysis of biomedical objects, determination of components in food products, and quick detection of explosives or drugs will be more actively created. Express analysis tools do not require significant costs and skilled performers. These are portable analytical instruments, including those of pocket type, sets of chemical or biochemical testtools-tubes, tablets, pieces of paper, etc. The simplicity, convenience, low cost of such tools should be combined with the quite acceptable reliability of the results obtained. The testing, production, and widespread use of portable systems for express analyses will undoubtedly be a clear trend. There are many companies in the world that specialize in this direction. Of course, for out-of-laboratory analysis, higher-level devices can also be used, e.g., in mobile laboratories, while providing the stability of equipment to the conditions of transportation and uncomfortable use.

There are great prospects for speciation analysis, i.e. recognition and quantification of various chemical and physical forms of existence of components of interest to the analyst. We are talking about the oxidation states of elements, their

*Corresponding author: Yu A Zolotov, Department of Chemistry, Lomonosov Moscow State University, 1/3 Leninskie gory, Moscow 119991, Russia

Accepted: November 10, 2020

Published online: November 12, 2020

Citation: Zolotov YA (2020) Some Prospects for Chemical Analysis. Chromatogr Spectrosc Tech 3(1):34-38 
various chemical compounds; about dissolved, colloidal, or sorbed forms, etc. While work is being done mainly with various forms of a relatively small number of elements-arsenic, tin, chromium, and others, with emphasis on oxidation states, the forms of which differ in toxicity, partly on the types of ions and complex compounds of elements [14]. Undoubtedly, the need for such research will only grow.

One can expect the development of direct analysis, i.e. non-destructive and without separation, primarily in the way of using physical phenomena. The concept of "non-destructive" naturally refers to solids, we now have examples of the direct determination of many components, almost exclusively elements; this is achieved by several, primarily spectroscopic, methods [15]. However, one must move forward; one of the tasks is to determine without destruction the state of oxidation of elements in solids; nowadays, the electron spin resonance method makes it possible to do something, some other methods, but this is not enough. We should hope that thought will work in this direction. Analysis without separation of components relates to liquid and gaseous objects. Some gas mixtures can already now be relatively easily analyzed without separation into components, e.g., by diode laser spectroscopy in the infrared range, however the general goal is such analysis relating to solutions, moreover, for components of a very different nature.

The tools of local analysis of solid objects require further development. Such an analysis is needed for material scientists, geochemists, forensic scientists, and many other specialists. This refers to point analysis, surface scanning [16] and evaluation of the distribution of components in depth. Many achievements of related sciences and various technical solutions are successfully used here. X-ray microanalysis, $X$-ray electron spectrometry (ESCA), Auger spectrometry, and variants of elemental mass spectrometry make it possible to solve many current problems, not always, however, with good accuracy. We have to look for new approaches, in particular to layer-by-layer analysis. Laser sampling is likely to play a role, including in laser-induced emission spectrometry.

Over time, remote analysis is likely to be used more often. Now these are variants of controlling processes with toxic and radioactive substances; analysis of environmental objects, especially air, using automatically operating stations, individual instruments and sensors, impressive analytical determinations in outer space and other. Various laser techniques are used for the purpose? For example laser indused breakdown spectrometry (LIBS) [17]. The number of remote analyses will grow with the spread of this methodology to new objects, to solve a wider range of tasks. To do this, original approaches, non-standard methods are needed, in particular, it is possible to use more actively the capabilities of fiber optics for transmitting laser radiation.

Among the "internal" trends in analytical chemistry, stimulated also by practical considerations, is the miniaturization of analysis [18-22]. It includes the development of small-sized instruments and devices and reduction in the mass and volume of analyzed samples. In some cases, miniaturization acts as an indispensable condition for solving the analytical problem. In addition, miniaturization, as one knows, is the way to save work space, energy, reagents, and valuable analyzed material. An example of miniaturization can be the wide use of micro-solid-phase extraction as a sample preparation method in chromatographic analysis [23]. As far as analytical instruments are concerned, a reduction in their size without a significant degradation of characteristics can be achieved for very many types of instruments, even for mass-spectrometers [24] or ESR-spectrometers. As an example, we can mention the gas microchromatographs. It is clear that this trend will continue in the future.

A separate topic is about the bright future of chemical sensors and their systems. However, the lack of a generally accepted definition of chemical sensors does not make it possible to draw a clear picture here. But it is clear that, say, gas sensors, including ay about semiconductor ones, solve important tasks of industrial control.

We need in the further automation of mass (routine) chemical analysis, especially on the basis of computerization. The two components of this process are automation of measuring instruments and automation of sample preparation systems. Automation will provide an increase in laboratory productivity, objectification of results, and saving labor costs. Automatization of laboratory wet analysis was successfully realized using flow injection analysis and related techniques. As to industrial analysis and control, different gas analyzers, industrial chromatographs and other instruments help to solve many tasks of automatization of the control [25].

The problem of identification of objects is solved by many methods, analytical ones are not the only ones, but they occupy an important place. There are many tasks here and many, as it seems, opportunities still unknown to anyone, e.g., on the way of using generalized indicators (sum parameters), chemometric methods of pattern recognition, and other approaches that make it possible not to perform component analysis. Technical and methodological solutions such as electronic nose or electronic tongue will help, again using chemometric methods.

One of the emerging and future directions in the development of analytical chemistry is its "greening". Green analytical chemistry is part of the green chemistry, moreover, already solidly formed. There are several books in the titles of which there is green analytical chemistry [26-28], the number of reviews and propaganda articles, not to mention the original works, is very large. There is no doubt that the struggle with the use of toxic solvents, with large volumes of harmful wastes, etc. will continue. In the publication by Polish analysts [29], twelve principles of green analytical chemistry, the implementation of which should be provided if possible, were formulated.

\section{Some General Approaches to Chemical Anal- ysis}

How the analytical chemistry will answer to these requirements? As before, a significant place will have to be occupied by the search and study of new phenomena, regularities, and properties of substances that can form the basis of fun- 
damentally new methods and tools of chemical analysis, including multicomponent and highly selective. This search involves expanding the list of areas of science and technology, the achievements of which can be used; in this list there are chemistry, physics, biochemistry, mathematics, and a number of areas of technology. For example, much more can be expected from physics-diode lasers, terahertz spectrometry, holography, etc.; from expanding and strengthening the already existing segment of analytical chemistry called nanoanalytics; from introducing into practice already developed nanoanalytical methods. Success in searching a fundamentally new can and should be fertilized by the collaboration of physicists-non-analysts or materials scientists with professional analysts.

When solving practical problems, for example problems of the analysis of environmental objects, wider use of multistage analysis with screening and rejection of samples at the first stages is possible [30,31]. At minimum, this saves a lot of time, labor, and the resource of measuring equipment. The development of metrology of chemical analysis and related areas remains on the agenda. This is the development of absolute determination methods or absolute variants of methods (without calibration), and improvement of graduation systems and methods for processing analysis results, including big data. Achievements of chemometrics will be, of course, more widen used.

\section{Analytical Techniques and Tools}

What specific analytical techniques will be particularly intensively developed in the coming years? One can only extrapolate today's trends; it is difficult to predict the appearance of new methods, however the search areas should be groped. Almost all current methods will be applied to one degree or another in the practice of analysis; the devil lies in this very "degree". Spectroscopic, or rather spectrometric, methods will, of course, be developed scientifically and widely applied in practice. This applies to X-ray methods, IR and Raman spectrometry, especially surface-enhanced Raman spectrometry [32], and to atomic emission analysis, despite competition from inductively coupled plasma mass spectrometry (MS-ICP), and partly to atomic absorption (AAS). In X-ray methods, the influence of the matrix composition and graduation remain the problem. Apparently, good prospects for the method with total external reflection. The use of synchrotron radiation for $\mathrm{X}$-ray spectral analysis remains exotic, although a "desktop synchrotron" has been created [33].

There is no clarity with the terahertz region. In this region of the spectrum, where the lines of weak intramolecular bonds lie, it is easier to identify organic molecules with slightly different groups. This makes it possible to detect explosive and toxic substances, including in packages through which radiation passes without noticeable attenuation. However, when using this area, noticeable difficulties must be overcome. Analysts should to a greater extent turn their eyes to the radio range (NMR, ESR, and NQR). These methods have significant analytical potential; e.g., examples of the use of NMR spectrometry and especially NQR for the detection of explosives are known [34].
Atomic absorption spectrometry will be used in applied laboratories, although not as intensively as, say, in the 1980s, and the number of publications using this method as such will apparently decrease further. A renaissance is possible on the path to multi-element AAS (there is an instrument from AnalytikJena Co.). Mass spectrometry, including (and maybe especially) in combination with chromatography, will be greatly advanced both on the pages of journals and in applied laboratories. We observed a very intensive development of the method over the past decades and its widespread use both on the leading edge of science (genomics, proteomics, and other "omics"), and in the practice of analyzing complex mixtures of industrial or other origin. Speaking about significance or prospects, we clearly separate these areas-the sciences and practices of analysis: if few publications are devoted to the method, this does not mean at all that it is not important for practice. And vice versa. In the case of mass spectrometry, a high score will be set in both areas. On perspectives of mass spectrometry see in more details in $[35,36]$.

There is practically no doubt in the happy future of modern chromatographic methods, of course, in the hybrid version, i.e. in close unity with low-selective determination methods implemented in the form of detectors. We are so accustomed to the very hybrid variant that we have already forgotten that chromatography itself is simply a separation method. Here it is necessary to make an assumption that the integration of methods and the creation of corresponding devices, i.e. the development of combined, hybrid (hyphenated) methods will occupy a significant place also in the future. Returning to chromatographic methods, constant appearance of new variants, in fact new methods, significantly expanding analytical capabilities (ion chromatography, hydrophilic, ultra-HPLC, tandem variants, new detectors, etc.) should be noted. Surely the development will continue actively.

It seems that we will see a further narrowing of the scale of practical use of electrochemical methods, despite the development of electrochemical sensors. These sensors, of course, are an interesting object of scientific research, but, it seems, are not yet very widely used in practice in spite of the development of perspective devices, for instance sweat based wearable sensors [37] and other. Research work on electrochemical methods will be, certainly, continued, and it is possible that we can expect the creation of fundamentally new electrochemical methods. Little can be said about nuclear-physical methods of analysis (radioanalytical techniques). Due to a change in society's attitude to the use of nuclear reactions, it is hardly possible to expect a return to interest in these methods in the near future, except perhaps gamma spectrometry, the study of natural radioactivity, and monitoring contaminated areas. Thermal methods of analysis are more interested in by inorganic chemists, material scientists, it is not by chance that derivatographs were used in laboratories of such specialists. Special prospects in these methods are not yet visible.

We have had tremendous success in recent decades in the field of sample preparation. Flow-injection analysis and its analogues, solid-phase extraction, numerous microvariants of liquid extraction, the use of microwave radiation, autoclaves, 
etc. can be named. These successes make it possible to believe that this area will continue to develop actively, including in dynamic variants, including in the continuous version.

\section{Objects of Analysis and Analytes}

Let us briefly settle on possible future priorities in the field of objects of analysis. The use of chemical analysis in medicine, primarily for the diagnosis of diseases, will be more and more significant. One of the tasks here is to find, together with medical workers, novel reliable markers of diseases in a non-invasive analysis, such as human secretions-expired air, saliva, tears, sweat, sebum and, of course, urine. A lot of research is being done in this direction; there are few reliable methods (not counting the methods of urine analysis), especially those that have been adopted into practice. There is reason to hope that diagnostic methods by the expired air will become routine in clinical practice in the future.

The analysis and control of other objects having a direct relationship to humans-food products, including drinking water, and medicines [38] are playing an increasingly important role. Of course, the analysis of well-established and very important objects-environmental objects, metals and alloys, mineral geological objects, oil, etc. will also be carried out in large-scale order. Key analytes will likely include components of biological fluids and tissues, vitamins, antibiotics, pesticides, explosives, dissolved oxygen, toxic gases, and some radioactive and toxic elements.

\section{Miscellaneous}

In almost all countries there is a dense network of analytical laboratories, in which tens of thousands of people work. Many laboratories belong to state services-sanitary-hygienic, agrochemical, ecological, geological, forensic, military-chemical, and hydrometeorological services. A large number of laboratories relate to enterprises, as well as universities, research institutes, etc. In methodological plan, these laboratories are poorly integrated, especially since the community of analysts is a difficult concept. Many physicists, biochemists, engineers, and other specialists, in the course of their work, become essentially analysts, often without realizing or recognizing this. When learning to work on complex, expensive devices, it is rational to create a system of computer simulators; such attempts have already been made, but device manufacturers have so far paid little attention to the creation of corresponding programs. Finally, it is desirable to take attention to the resent interesting article by $\mathrm{F}$. Adams and $\mathrm{M}$. Adriaens on modern analytical chemistry and its perspectives [39].

\section{References}

1. Ho TD, Zhang C, Handao LW, et al. (2014) lonic liquids in analytical chemistry: Fundamentals, advances, and perspectives. Anal Chem 86: 262-265.

2. McLennan F (1995) Process analytical chemistry. In: McLennan F, Kowalski BD, Process Analytical Chemistry. Springer, Heidelberg, 1-13.

3. Carey WP, Burgess LW (1995) Future developments of chemical sensors and analyzer systems. In: McLennan F, Kowalski BD, Process analytical chemistry. Springer, Heidelberg, Germany,
353-355.

4. Mitra S, Brukh R (2003) Sample preparation: An analytical perspectives. Sample preparation techniques in analytical chemistry, New York, John Wiley and Sons 162.

5. Spietelun A, Marcinkowski L, de la Guardia M (2013) Recent development and future trends in solid phase microextraction techniques towards green analytical chemistry. J. Chromatogr A 1321: 1-13.

6. Yamini Ya, Seidi Sh, Rezazadeh M (2014) Electric field-induced extraction and separation techniques: promising trends in analytical chemistry. Anal Chim Acta 814: 1-22.

7. Wu Yao, Belmonte I, Sykes KS, et al. (2019) Perspectives on the future role of aptamers in analytical chemistry. Anal Chem 91: 15335-15344.

8. Cui Li, Zhang DanDan, Zhu Yong Guan (2019) Perspectives on surface-enchanced Raman spectroscopic investigation of microbial wordl. Anal Chem 91: 15345-15354.

9. Kulesza PJ, Cox JA (1998) Solid-state voltammetry-analytical prospects. Electroanalysis 10: 73-80.

10. Giakoumaki A, Melessanaki K, Anglos D (2007) Laser-induced breakdown spectroscopy (LIBS) in archeological science-applications and prospects. Anal Bioanal Chem 387: 749-766.

11. Blasco C, Pico Y (2009) Prospects for combining chemical and biological methods for integrated environmental assessment. Trends Anal Chem 28: 745-757.

12. Zolotov Yu A (2011) On some trends. Talanta 85: 2249-2250.

13. Vnelaboratornii Khimicheskii Analiz (2010) Out-of-Laboratory chemical analysis. Zolotov Yu A Moscow, Nauka Publ House, 564.

14. Yu Xiaoping, Liu Chenglong, Guo Yafei, et al. (2019) Speciation analysis of trace arsenic, mercury, selenium and antimony in environmental and biological samples based on hyphenated techniques. Molecules 24: 926.

15. Borgwardt TC, Wells DP (2017) What does non-distructive analysis mean? Cogent Chemistry.

16. Sykes D (2017) Surface chemical analysis. In: Kasap S, Capper $P$, Springer handbook of electronic and photonic materials, Springer Handbooks, Cham, Springer.

17. Miziolek AW, Palleschi V, Schechter I (2009) Laser Induced Breakdown Spectroscopy. Cambridge Univ Press.

18. Hussain Ch M (2020) Handbook on miniaturization in analytical chemistry. Application of Nanotechnology. Amsterdam, Elsevier 364.

19. Pena Pereira F (2014) From conventional to miniaturized analytical systems. In: Miniaturization in sample preparation, 1-28.

20. Rios A, Escarpa A, Simonet B (2009) Miniaturization of analytical chemistry. New York, John Wiley and Sons.

21. Janasek D, Franzke J, Manz A (2006) Scaling and the design of miniaturized chemical-analysis systems. Nature 442: 374-380.

22. Vysetti B (2016) Current advances in the miniaturization of chemical instruments-Application in cosmochemistry, geochemistry, exploration and environmental sciences. Spectroscopy 31 : 40-44.

23. Pawliszyn Ja (2002) Solid phase microextraction. In: Pawliszyn Ja, Sampling and Sample Preparation for Field and Laboratory, Amsterdam, Elsevier 389-477. 
24. Medina DAV, Maciel EVS, Lancas FM (2020) Miniaturization of liquid chromatography coupled to mass spectrometry. 3. Achievements on chip-based LC-MS devices. Trends Anal Chem 131: 116603.

25. Fleischer H, Thurow K (2019) Theory and practical application of automatic solutions in analytical measurements. American Laboratory.

26. De la Gardia M, Garrigues S (2020) Past, present and future of green analytical chemistry. In: Challenges in Green Analytical Chemistry, 2: 1-18.

27. De la Gardia M, Garrigues S, Handbook of green analytical chemistry. New York, John Wiley and Sons.

28. Plotka Wasylka Ju, Namesnik Ja (2020) Green analytical chemistry. Past, Present and Perspectives. Cham, Springer Nature 450.

29. Galuszka A, Migaszewski Z, Namiesnik Ja (2013) The 12 principles of green analytical chemistry and the significance mnemonic of green analytical practices. Trends Anal Chem 50: 78-84.

30. Valcarcel M, Cardenas S, Gallego M (1999) Sample screening systems in analytical chemistry. Trends Anal Chem 18: 685-694.

31. M Valcárcel, SCárdenas, Mgallego (2004) Screening analysis: An overview of methods applied to environmental, clinical and food analysts. Trends Anal Chem 23: 203-216.
32. Langer Ju, De Aberasturi DJ, Aizpurua Ja, et al. (2020) Present and future of surface enhanced Raman scattering. ACS Nano 14: 28-117.

33. Kumakhov MA (2004) X-Ray and capillary optics. Proceedings SPIE 5943: 102-115.

34. Itozaki H (2009) Nuclear quadrupole resonance for explosives detection. In: Furuta K, Ishikawa J, Anti personnel landmine detection for humanitarian demining. London, Springer, 147-155.

35. Trevorrow P, Kilgour DPA (2018) Perspectives on the future of mass spectrometry. Rapid Commms Mass Spectrometry 33: 1-2.

36. Graca DC, Lescuyer P (2019) Ultrahigh performance mass spectrometry in clinical chemistry: A taste of the future? Clinical Chemistry 65: 943-945.

37. Mohan AMV, Rajendran V, Mishra RK, et al. (2020) Resent advances and perspectives in sweat based wearable electrochemical sensors. Trands Anal Chem 131: 116024.

38. Bakirhan NK, Ozkan SA (2020) Resent advances and future perspectives in pharmaceutical analysis. Current Pharmas. Analysis 16: 1.

39. Adams F, Adriaens M (2020) The metamorphosis of analytical chemistry. Anal Bioanal Chem 412: 3525-3537. 